\title{
TWO-WEIGHT ENTROPY BOUNDEDNESS OF MULTILINEAR FRACTIONAL TYPE OPERATORS
}

\author{
Mingming CAO And Qingying Xue
}

Abstract. This paper will be devoted to study the two-weight norm inequalities of the multilinear fractional maximal operator $\mathscr{M}_{\alpha}$ and the multilinear fractional integral operator $\mathscr{I}_{\alpha}$. The entropy conditions in the multilinear setting will be introduced and the entropy bounds for $\mathscr{M}_{\alpha}$ and $\mathscr{I}_{\alpha}$ will be given.

Mathematics subject classification (2010): 26D10.

Keywords and phrases: Two-weight, entropy conditions, Carleson embedding theorem, multilinear fractional integral operator.

\section{REFERENCES}

[1] M. CAO, K. Li, Q. XuE, A Characterization of two weight norm inequality for Littlewood-Paley $g_{\lambda}^{*}$-function, http://arxiv.org/abs/1504.07850.

[2] M. CAO, Q. XUE, Characterization of two-weighted inequalities for multilinear fractional maximal operator, Nonlinear Analysis: TMA 130 (2016) 214-228.

[3] M. CAO, Q. Xue, K. YABUTA, On multilinear fractional strong maximal operator associated with rectangles and multiple weights, Rev. Mat. Iberoamericana., to appear.

[4] W. Chen, W. Damián, Weighted estimates for the multilinear maximal function, Rend. Circ. Mat. Palermo. 62 (2013), 379-391.

[5] S. Chen, H. Wu, Q. XuE, A note on multilinear Muckenhoupt classes for multiple weights, Studia Math. 223 (1) (2014).

[6] T. HÄNninen, T. Hytönen, K. LI, Two-weight $L^{p}-L^{q}$ bounds for positive dyadic operators: Unified approach to $p \leqslant q$ and $p>q$, Potential Anal., 45 (2016), 579-608.

[7] T. HYTÖNEN, The two-weight inequality for the Hilbert transform with general measures, http://arxiv.org/abs/1312.0843v1.

[8] T. Hytönen, Representation of singular integrals by dyadic operators, and the $A_{2}$ theorem, http://arxiv.org/abs/1108.5119v1.

[9] T. HYTÖNEN AND C. PÉREZ, Sharp weighted bounds involving $A_{\infty}$, Analysis and PDE. 6 (2013), no. 4, 777-818.

[10] M. T. LACEY, The two weight inequality for the Hilbert transform: a primer, submitted (2013), http://www.arxiv.org/abs/1304.5004.

[11] M. T. LACEY, Two weight inequality for the Hilbert transform: a real variable characterization, II, Duke Math. J. 163 (2014), no. 15, 2821-2840.

[12] M. T. LACEY, K. LI, Two weight norm inequalities for $g$ function, Math. Res. Lett. 21 (2014), no. 03, 521-536.

[13] M. T. LACEY, K. LI, On $A_{p}-A_{\infty}$ type estimates for square functions, Math. Z. 284 (2016), 12111222.

[14] M. T. LACEy, E. T. SAWyer, I. URIARTE-Tuero, Two weight inequalities for discrete positive operators, http://arxiv.org/abs/0911.3437v4.

[15] M. T. Lacey, E. T. SAWYer, I. URiarte-Tuero And C.-Y. Shen, Two weight inequality for the Hilbert transform: a real variable characterization, I, Duke Math. J. 163 (2014), no. 15, 2795-2820.

[16] M. LACEY AND S. SPENCER, On entropy bumps for Calderón-Zygmund operators, Concr. Oper. 2015, 2: 47-52. 
[17] K. LI, K. Moen, W. Sun, Sharp weighted inequalities for multilinear fractional maximal operator and fractional integrals, Math. Nachr. 288 (2015), no. 5-6, 619-632.

[18] K. LI, W. SUn, Characterization of a two weight inequality for multilinear fractional maximal operators, Houston J. Math. 42 (2016), no. 3, 977-990.

[19] K. LI, W. SUN, Two weight norm inequalities for the bilinear fractional integrals, Manuscripta math. 150, 159-175 (2016).

[20] F. Nazarov, S. Treil And A. Volberg, The Bellman function and two weight inequalities for Haar multipliers, J. Amer. Math. Soc. 12 (1999), no. 4, 909-928.

[21] R. RAHM, S. SPENCER, Some entropy bump conditions for fractional maximal and integral operators, Concr. Oper. 2016, 3: 112-121.

[22] E. SAWYER, A characterization of a two weight norm inequality for maximal operators, Studia Math. 75 (1982), 1-11.

[23] E. SAWYER, A two weight weak type inequality for fractional integrals, Trans. Amer. Math. Soc. 281 (1984), 339-345.

[24] E. SAWYER, A characterization of two weight norm inequalities for fractional and Poisson integrals, Trans. Amer. Math. Soc. 308 (1988), 533-545.

[25] B. F. SEhbA, On two-weight norm estimates for multilinear fractional maximal function, http://arxiv.org/abs/1502.01612.

[26] S. TReil And A. VolberG, Entropy conditions in two weight inequalities for singular integral operators, Adv. Math. 301 (2016) 499-548. 